\title{
Energy and Transmission Allocation in the presence of Overlapping Electricity Markets
}

\author{
Adamantios Marinakis \\ Anastasios G. Bakirtzis, Senior Member, IEEE \\ Thierry Van Cutsem, Fellow, IEEE
}

\begin{abstract}
The possibility for market participants to simultaneously place their bids in different markets across an interconnection is investigated in this paper. Transaction schedulers settle multilateral transactions among participants, while a central entity coordinates the overall operation through interactions with the transaction schedulers. Two issues are dealt with in this context. First, the market participants are allowed to place their bids simultaneously in more than one transactions scheduler's market, and, second, the available transmission capacity is fairly shared among the transaction schedulers. Economically interesting transactions are favored, while confidentiality of market data and independence of transaction schedulers' clearing mechanisms are preserved. The corresponding iterative algorithm is illustrated in detail on a 15-bus as well as the IEEE-RTS system.
\end{abstract}

Keywords: Electricity market, market clearing, congestion management, market coupling, overlapping markets.

\section{INTRODUCTION}

\section{A. Existing situation}

In modern power systems, several areas, controlled by separate entities, form altogether larger interconnections inside which electricity is traded [1]. In Europe, for instance, the areas correspond to countries and the entities to Transmission System Operators (TSOs). While a lot of research effort has been devoted to improving electricity markets inside areas, comparatively less attention has been paid to organizational structures and algorithms allowing separate areas to be operated in a seamless way in terms of inter-area electricity trade.

Long-term forward contracts between different areas have been in practice even before the liberalization process. This paper, however, focuses on the operation of spot markets, from day-ahead up to real-time, and the development of algorithms to facilitate the inter-area trade.

A typical way to do so has been the posting by TSOs of Available Transfer Capacity (ATC) values for importing and/or exporting at each interconnection and the selling of consistent transmission rights to the market actors. This is referred to as explicit auction of transmission capacity, since the latter is auctioned and sold separately from energy. In such a framework, for instance, a broker purchases export and import rights from the areas where the generator and the consumer respectively are located, in this way settling an interarea transaction. Explicit auction is currently the prevailing allocation mechanism of scarce interconnection resources in

A. Marinakis (marinakis@montefiore.ulg.ac.be) is Ph.D. student in the Dept. of Electrical Engineering and Computer Science (Montefiore Institute) of the University of Liège, Sart Tilman B37, B-4000 Liège, Belgium.

T. Van Cutsem (t.vancutsem@ulg.ac.be) is a research director of FNRS (Fund for Scientific Research) and adjunct professor in the same department.

A.G. Bakirtzis (bakiana@eng.auth.gr) is professor in the Dept. of Electrical and Computer Engineering of the Aristotle University of Thessaloniki, Greece.
Europe. Although attractive in theory, this approach has been found in practice to yield some inefficient use of the network. The main reasons are: it is difficult for the participants to anticipate what the value of each transmission line will be for them, some participants tend to hoard capacity that they don't finally use, and pancaking of allocations appears when several borders are involved in a transaction [2].

The alternative, increasingly used in the last years, is implicit auction for congestion management, where the use of the transmission system is allocated implicitly at the time the energy market is cleared [3]. This is the main way intraarea congestion management is treated in some parts of North America, with the several pool based Locational Marginal Pricing (LMP) approaches [4]. Another implicit auction approach, called market splitting, has been used for years in the Nordic market (Scandinavian countries) where in case of congestion the market is split in two or more price areas [5].

It seems that implicit auction is the future (and already the present in some cases) way of managing cross-border transmission capacities in Europe. The prevailing mechanism for doing so is the so-called market coupling. Both the LMP and the market splitting approaches require a centralized market operator that combines the bids in a market clearing procedure. On the other hand, market coupling is an implicit auction similar to market splitting but performed in reverse order. First, each sub-market is cleared; then, these markets are coupled. It is thus a method performing coordination among different markets, each using its own rules inside its area [6].

The first implementation of this approach was the Trilateral Market Coupling (TLC) in operation since 2006 between France, Belgium and the Netherlands. It is organized as a decentralized, multilateral contractual arrangement between the following participants [3]. The Power Exchanges (PXs), namely APX, Belpex and Powernext, who provide the IT systems and run the common coupling algorithm, and, the TSOs, namely RTE, Elia and TenneT, who calculate cross border capacities, set up physical exchanges, share congestion revenues and pay the market coupling service fee that is determined locally. Regulatory oversight remains with the national regulators and/or is subject to national legislation.

A detailed description of the TLC algorithm can be found on the Web sites of the above PXs (e.g. [2]). Basically, it consists of each market participant bidding in the day-ahead market of the area where it is physically located, using the rules and IT tools of the corresponding PX. These (sell or purchase) bids are used by the PXs to construct the net export curve of their markets, i.e. the difference between total sales and total purchases of this market as a function of the Market 
Clearing Price (MCP). These curves are assembled in the central coordination module so that markets with the highest MCPs import electricity from markets with the lowest MCPs. In the absence of congestion, the result is an import/export pattern between markets in such volumes that the three local MCPs become equal. Otherwise, import/export is settled up to the ATCs and the markets end up with different prices. This mechanism enables local markets participants to "see" a larger liquidity, not limited to their area, within the limits of the cross-border capacities of course.

The extension of TLC to the five countries of the Central Western Europe (CWE) region has been announced for 2010. This involves Germany and Luxemburg in addition to the three TLC countries. A more sophisticated algorithm is envisaged [7], although it retains the ideas that a market participant interacts only with the PX of its area, while some central calculations take care of energy being exported from low to high price areas, within the limits of transfer capacity. First, an ATC-based modeling of the network constraints will be used, but it is planned to switch soon to a more precise flowbased network model, in which critical branches (tie- and some internal lines) will be defined by the CWE TSOs. For the time being, the above market coupling mechanisms apply to dayahead procedures only. Steps are also taken towards opening intra-day and real-time markets to foreign players [8].

The above outlined trilateral, and soon pentalateral, initiative couples the markets of the involved PXs. It should be noted, however, that these PXs do not involve but a fraction of the spot energy trade in Europe, where trading arrangements are mainly bilateral. Most of the wholesale trade is in the Over-The-Counter markets, often supplemented with day-ahead auction trade organized by the national PXs [9]. The advantage of having the PXs organizing these auctions is that they use simple rules to settle contracts at a point of time where it is not worth getting into time consuming negotiations. Power Exchanges are also counter-party for all transactions so that trade is anonymous and traders do not have to worry about counter-party risk. However, it could also be argued that PXs are not strictly necessary market components [9]. Still, most European countries have a PX often as a result of private initiatives. The PXs often do not have to take network constraints into account at all, or they do only partly.

Compared to Europe, the North American wholesale markets appear more weakly linked, if at all. As considered in [9], [10], it may be more difficult to couple these markets because they apply a different implementation of nodal pricing, making it practically difficult to harmonize the handling of network constraints; even more if the latter is already fine-tuned, which is less the case in Europe. There is, however, a common market initiative between MISO and PJM who are working towards the development of complementing system operations and one robust, non-discriminatory wholesale electricity market to meet the needs of all customers and stakeholders [11].

\section{B. Scope of the paper}

Before going into details, it is appropriate to formalize the framework of the proposed approach, since there are various electricity market implementations. We call System Operator (SO) the entity responsible for operating the transmission system of a particular area, while we call Transaction Scheduler (TS) every entity responsible for settling transactions between producers and consumers. For instance, a PX is a TS, but other entities also fit the description, such as a broker who settles bilateral or multilateral transactions. The SO is typically a TS when dealing with real-time operation (balancing market, generation re-dispatch, etc.).

Usually, there exists one PX (or none) per area, but in principle nothing prevents several PXs from co-existing and competing within an area. On the other hand, a PX can extend its activities over more than one area. This is going to happen in a near future with the merging of Powernext and EEX (French and German PXs, respectively).

This paper investigates whether the constraint that a wholesale market participant should be part of a particular spot market, defined by its geographic location, could be relaxed. Thus, the presented approach assumes that every market participant is allowed to bid in whatever market (represented by a TS), irrespective of where it is located. More generally, the paper proposes a framework and an algorithm to let market actors use the grid in a coordinated way to perform commerce of electrical energy without them being constrained to do so via a TS covering only their geographic location.

Clearly, the idea that any market participant may place its bids in the market of any TS operating in the interconnection would result in the appearance of "overlapping markets" and would make inter-area congestion management even more important. The development of a coordinating framework is thus required. This framework should enable free spot trade of electricity. The TSs should be able to compete freely first to attract market participants interested in settling transactions and second to obtain transmission capacity in order to support these transactions.

Furthermore, this work is based on the assumption that the SOs of an interconnection are willing to co-operate in the setting up of a common model of the grid and to delegate part of the congestion management tasks to a central coordinating entity. These assumptions seem acceptable and go with the present trend, at least in Europe [12].

The proposed approach offers a decentralized way of coordinating multilateral transactions. In this spirit, Ref. [13] proposes a new operating paradigm in which the decision mechanisms regarding economics and reliability (security) of system operation are separated. In [14], the author proposes two decentralized procedures in which each Regional Transmission Organization (RTO) administers its energy market and also acts as a transmission coordinator to achieve feasible and efficient use of congested transmission by all markets in the interconnection. Participants in any RTO market are allowed to schedule transactions into, out of, or across any RTO control areas. The resulting overlapping markets are modeled, while, since when transmission capacity is limited markets compete for the use of the limited transmission paths, two methods for allocating this capacity are proposed. Closely related is also the work in [15], which proposes a decentralized model for dc load flow based congestion management for the forward 
markets via optimal resource allocation.

The approach developed in the present paper considers the following two prerequisites:

1) Transparency of the grid data: SOs are responsible for constructing a common model of the grid and make it available to all participants. In this way everybody will be able to check that the coordinating computations made by the SOs are fair.

2) Confidentiality of each market data and procedures: the TSs should not be asked to provide any intermediate information of their market clearing procedures. They should only announce their final schedules and prices.

The paper is organized as follows. The problem is stated in Section II, which points out energy and transmission allocation issues, and outlines the proposed approaches. In Section III the procedure is presented in detail, while its features are highlighted in Sections IV and V using illustrative examples. Additional aspects are discussed in Section VI, while the paper ends with a conclusion in Section VII.

\section{STATEMENT OF THE PROBLEM AND OUTLINE OF THE APPROACH}

\section{A. Market clearing and transmission system modeling}

Let $M$ be the number of TSs. Each TS clears the market it represents, using its own rules. The outcomes are scheduled generation-load quantities together with the corresponding prices offered to each generator or asked by each load.

Although the clearing may be implemented in various ways, we formalize it as an optimization problem. For the $m$-th TS, this optimization takes on the form :

$$
\begin{aligned}
& \min _{p_{g_{i}}^{m}, p_{l_{j}}^{m}} \sum_{i}\left\{c_{i}^{m} p_{g_{i}}^{m}\right\}-\sum_{j}\left\{b_{j}^{m} p_{l_{j}}^{m}\right\} \\
\text { s. t. } \quad & \sum_{i} p_{g_{i}}^{m}=\sum_{j} p_{l_{j}}^{m} \\
0 & \leq p_{g_{i}}^{m} \leq p_{g_{i}}^{\text {max }, m} \\
0 & \leq p_{l_{j}}^{m} \leq p_{l_{j}}^{\text {max }, m}
\end{aligned}
$$

where $c_{i}^{m}$ (respectively $b_{j}^{m}$ ) is the bid of generator $i$ (consumer $j$ ) in market $m, p_{g_{i}}^{m}\left(p_{l_{j}}^{m}\right)$ is the allocated quantity of generator $i$ (consumer $j$ ), $p_{g_{i}}^{\max , m}$ is the maximum power that generator $i$ is willing to produce for market $m$, and $p_{l_{j}}^{\max , m}$ is the maximum power that load $j$ is willing to consume. Equation (2) expresses that each TS has a balanced schedule.

The net power injection at bus $k$ scheduled by the $m$-th TS is given by:

$$
p_{k}^{m}=\sum_{i \in k} p_{g_{i}}^{m}-\sum_{j \in k} p_{l_{j}}^{m}
$$

This is written in vector form as $\mathbf{p}^{m}=\mathbf{p}_{g}^{m}-\mathbf{p}_{l}^{m} \quad$ where vectors $\mathbf{p}_{g}^{m}$ and $\mathbf{p}_{l}^{m}$ have zeros in the entries corresponding to buses with respectively no generation and no load allocated by the $m$-th TS.

The vector of net bus power injections is obtained as the summation of all the TS schedules:

$$
\mathbf{p}=\sum_{m} \mathbf{p}^{m}=\sum_{m} \mathbf{p}_{g}^{m}-\sum_{m} \mathbf{p}_{l}^{m}
$$

Once this vector is known, branch power flows can be computed using a model of the entire network. In this paper a DC model of the interconnection is used. This is a commonly used model in market clearing problems and it is well suited to the linear computations presented in the remaining of the paper. It is assumed that the various SOs in the interconnection assemble and share such a network model, which they use to coordinate the overlapping markets simultaneous clearings.

Let $B$ be the number of branches and $N$ the number of buses in the system. In order to assess the impact of the power injection schedule on branch flows, we resort to well-known Power Transfer Distribution Factors (PTDF). Let $F_{b}^{k l}$ be the fraction of a transaction from bus $k$ to bus $l$ that flows over branch $b(k, l=1, \ldots, N ; b=1, \ldots, B)$. According to [5]:

$$
F_{b}^{k l}=\frac{X_{i k}-X_{j k}-X_{i l}+X_{j l}}{x_{b}}
$$

where $i$ and $j$ are the terminal buses of the branch, $x_{b}$ its reactance, $X_{i k}$ the entry in the $i$-th row and $k$-th column of the $N \times N$ bus reactance matrix $\mathbf{X}$, and similarly for the other entries. Assuming that bus $N$ is the slack bus, the $N$-th row and the $N$-th column of $\mathbf{X}$ have all zero elements [16].

The effect of the power injection $p_{k}$ at bus $k$ on the power flow of branch $b$ can be seen as the effect of a transaction $p_{k}$ between bus $k$ and the slack bus $N$. The power flowing through branch $b$ is thus given by:

$$
f_{b}=\sum_{k=1}^{N} F_{b}^{k N} p_{k}
$$

This is easily written in matrix form as :

$$
\mathbf{f}=\mathbf{S} \mathbf{p}
$$

where $\mathbf{f}$ is the vector of branch power flows and $\mathbf{S}$ is the $B \times N$ matrix relating branch power flows to bus power injections, and defined by:

$$
(\mathbf{S})_{b k}=F_{b}^{k N} \quad b=1, \ldots, B ; k=1, \ldots, N
$$

The choice of the slack bus influences the elements of $\mathbf{S}$. However, when assessing the contribution of the market schedules to branch flows, formula (9) will be applied to the injection vector $\mathbf{p}$ whose components sum up to zero, owing to (2), (5) and (6). Therefore, the net power injection caused by the $m$-th market at the slack bus is zero. Thus, the branch flows computed in (9) do not depend on the choice of the slack bus (losses being neglected in this approach).

As long as there is enough reactive compensation to keep voltage magnitudes constant at all buses, PTDFs have been shown to remain practically unchanged as the pattern of injections changes the loading of branches [17], [18], [19].

\section{B. Emerging issues}

Clearing the above mentioned overlapping markets without any concern for the grid flows is very likely to end up in branch overloads. The problem is not solved even if, following the idea proposed in [20], TSs are asked to include in their clearing problems (1-4) branch flow constraints of the type:

$$
-\mathbf{f}^{\max } \leq \mathbf{S}\left(\mathbf{p}^{m}+\sum_{m^{-}} \mathbf{p}^{m^{-}}\right) \leq \mathbf{f}^{\max }
$$


where $m^{-}$denotes all TS markets but the $m$-th one. Indeed, this constraint means that a TS will come up with a schedule that does not cause branch limit violation, given the last schedule announced by the other TSs. However, since the other TSs are clearing their own markets at the same time, the combined schedule $\mathbf{p}$ may quite well lead to overloads. The branch flow limits cannot be enforced by acting on each market irrespective of what the other markets are doing; instead, a coordinated congestion management is required.

Another issue has to do with the risk for the final schedule to be far from what could be reached by optimizing the whole system as a single market. The reason is that some attractive market participants (e.g. cheap generators), having placed their bids in a market, may be excluded when the latter is cleared, and thus remain inactive while they could still be used by another TS to reach a better schedule. One could argue that such a case should not persist in the long term, because market participants will "find their place". However, the problem will definitely appear in the short term. Hence, a mechanism should allow efficient shifting of participants between the various TS markets.

\section{Outline of the proposed approach}

We outline hereafter the solutions further developed in this paper to deal with the above two issues.

Regarding the congestion management issue, the proposed approach consists in sharing between the TSs the capacity of the most used branches so that the $m$-th TS, when clearing its market, would obey reduced flow limits $\mathbf{f}_{o v}^{\max , m}$, where the lowerscript $o v$ denotes the set of overloaded branches. The modified limits are such that $\sum_{m} \mathbf{f}_{o v}^{\max , m}=\mathbf{f}_{o v}^{\max }$, and are iteratively adjusted to the schedules announced by the TSs.

Regarding the issue of attractive participants staying inactive, the proposed solution consists in allowing market participants to place their bids in more than one market simultaneously. After the market clearings, the participant should be allocated to the TS from which it received the best offer (the highest price to be paid if it is a generator, or the lowest price to pay if it is a consumer). Price is assumed to be the criterion used to eventually select which TS a particular participant will be assigned to.

Both treatments suggest the presence of a coordinating entity. As to the congestion management issue, the coordinator will iteratively communicate to the TSs their corresponding reduced branch limits, which it will compute based on an agreed policy. As for the market participant allocation, the coordinator will check the prices offered by the TSs and announce the generation/load quantities finally allocated to each TS.

This coordinator may result from the joint efforts of the involved SOs. Its role is to facilitate electricity trading, while respecting the confidentiality of the TS data and the independence of their procedures. In this respect, the only information provided by the TSs to the central coordinator are their power injection schedules and the corresponding prices offered to the market participants.

\section{THE PROPOSED ALGORITHM}

Both coordinator tasks are now considered in detail, before describing the whole algorithm.

\section{A. Energy Allocation}

An iterative procedure, referred to as "Energy allocation loop", is implemented by the coordinator to allow the previously mentioned simultaneous dispatching of the market participants by all the TSs.

The procedure starts with the market participants placing their bids, each consisting of a maximum quantity (corresponding to available generation or to load asking to be served) and one price per TS ${ }^{1}$.

The TSs compete with each other trying to allocate in their final dispatch the most interesting participants. Thus, after having cleared its market, the $m$-th TS communicates to the coordinator its demanded bus generation vector $\mathbf{p}_{g}^{m, d e m}$ and consumption vector $\mathbf{p}_{l}^{m, d e m}$, together with the corresponding price vectors $\boldsymbol{\pi}_{g}^{m}$ and $\boldsymbol{\pi}_{l}^{m}$.

For a given generator $i$, if the total power demanded by the various TSs is below (or equal to) its capacity, i.e. $\sum_{m} p_{g_{i}}^{m, d e m} \leq p_{g_{i}}^{\max }$, that power is simply allocated to the various TSs as they requested. Otherwise, there is a conflict, and the role of the coordinator is to take care that the generator is finally dispatched at the most profitable possible prices. To this purpose, the coordinator allocates the power to one or several of the involved TSs by decreasing order of offered price. In case several TSs compete for the same generator with equal offered prices, the available power is shared in proportion with the requested quantities.

Hence, generally, some TSs will be left with power imbalances, and the markets have to be cleared again. In order the power just allocated to a TS not to be available to the others, the coordinator communicates reduced bounds $p_{g_{i}}^{\max , m}$ to the latter TSs.

Thus, the TSs come up with new demanded quantities and offered prices. At this stage, the coordinator repeats the above procedure, with the following two additional rules:

1) what was previously allocated to a TS and is still requested remains with that $\mathrm{TS}$;

2) what was previously allocated to a TS and is not requested any longer is made right away available to the other TSs.

These iterative adjustments lead to a gradual allocation of all demanded generations. Loads are handled in a similar way, but with the allocation performed by increasing order of prices requested by the TSs in order to serve them.

The procedure terminates when each market is balanced, no TS has incentive to further improve its schedule by dispatching available generation or load, and no conflict is left for any resource.

Note that no market participant is obliged to participate in the Energy allocation procedure. Indeed, a market participant may prefer to place its bid directly in a TS market because

\footnotetext{
${ }^{1}$ why market participants could bid differently to different TSs will be discussed in the sequel
} 
of a beneficial arrangement made with this TS or because it believes the announcement of the clearing price by the TS would unveil its bid. Furthermore, no TS is obliged to accept such bids. However, a TS may be willing to receive bids from the above described Energy allocation procedure owing to the risk of being left without enough participants interested in placing their bids in its market. Thus, what has been described refers to participants and TSs who choose to take advantage of the higher liquidity offered by the proposed mechanism.

Note also that different markets may impose different obligations or offer different benefits to their participants, which can make the prices that a participant receives from the various TSs for the same amount of energy not directly comparable with each other. This will be generally reflected on the individual price a market participant offers to each TS in its bid. Additionally, a predefined correction term can be applied when prices are compared by the central coordinator. This is easily incorporated in the presented procedure. Further discussion of this issue can be found in [21].

\section{B. Transmission allocation}

At the end of the Energy allocation procedure described above, the bus injection vector defined in (6) is available. Note that in general this vector also includes power injections that result from a bilateral agreement between parties, and hence have not been determined iteratively as described in the previous section.

The corresponding branch flows can be computed. If no limit is violated, the TS schedules can be approved. Otherwise, congestions are managed through an iterative procedure, referred to as "Transmission allocation loop", as detailed hereafter.

Assume that the power flow $f_{b}$ in the $b$-th branch $(b=$ $1, \ldots, B)$ exceeds its upper limit:

$$
f_{b}=f_{b}^{o} \geq f_{b}^{\max }>0
$$

Using Eqs. (6) and (9), this inequality can be rewritten as:

$$
\sum_{m} \mathbf{s}_{b} \mathbf{p}_{o}^{m} \geq f_{b}^{\max }
$$

where $\mathbf{s}_{b}$ is the $b$-th row of the $\mathbf{S}$ matrix and $\mathbf{p}_{o}^{m}$ is the schedule of the $m$-th TS, obtained as described in the previous section. It turns out that $\mathbf{s}_{b} \mathbf{p}_{o}^{m}$ is the participation of the $m$-th TS in the $b$-th branch flow. Obviously, all TS participations add up to the actual branch flow $f_{b}$.

From there on, the $m$-th TS is required to change its schedule from $\mathbf{p}_{o}^{m}$ to a new value $\mathbf{p}^{m}$ so that its contribution to the branch flow $f_{b}$ is decreased by at least a specified amount $\Delta f_{-}^{m}$ :

$$
\mathbf{s}_{b}\left(\mathbf{p}^{m}-\mathbf{p}_{o}^{m}\right) \leq-\Delta f_{-}^{m}
$$

with the sum of all $\Delta f_{-}^{m}$ values being equal to the branch overload to be corrected:

$$
\sum_{m} \Delta f_{-}^{m}=\sum_{m} \mathbf{s}_{b} \mathbf{p}_{o}^{m}-f_{b}^{\max }
$$

If both (14) and (15) hold true, the power flow will become:

$$
\sum_{m} \mathbf{s}_{b} \mathbf{p}^{m} \leq \sum_{m} \mathbf{s}_{b} \mathbf{p}_{o}^{m}-\sum_{m} \Delta f_{-}^{m}=f_{b}^{\max }
$$

and it will be decreased below or at its limit.

Similarly, if the branch overload has the opposite sign, i.e.

$$
f_{b}=f_{b}^{o} \leq-f_{b}^{\max }<0
$$

the $m$-th TS is required to change its schedule so that its contribution to the branch flow $f_{b}$ is increased by at least a specified amount $\Delta f_{+}^{m}$ :

$$
\mathbf{s}_{b}\left(\mathbf{p}^{m}-\mathbf{p}_{o}^{m}\right) \geq \Delta f_{+}^{m}
$$

with the sum of all $\Delta f_{+}^{m}$ values being equal to:

$$
\sum_{m} \Delta f_{+}^{m}=-f_{b}^{\max }-\sum_{m} \mathbf{s}_{b} \mathbf{p}_{o}^{m}
$$

which leads to bringing the power flow above or at its limit.

Thus, each TS is required to incorporate the constraints (14) and (18) into its market clearing problem (1-4). In fact, (14) can be rewritten as:

$$
\mathbf{s}_{b} \mathbf{p}^{m} \leq \mathbf{s}_{b} \mathbf{p}_{o}^{m}-\Delta f_{-}^{m}
$$

where the left-hand side represents the flow produced in branch $b$ by the schedule of the $m$-th TS, and the right-hand side can be interpreted as a reduced capacity allocated to that TS. A similar comment applies to (18).

As can be seen, the overload correction is shared among the various TSs and, unlike (11), the constraint handled by the $m$-th TS involves its own control variable $\mathbf{p}^{m}$ only.

Three aspects of the above procedure are now considered in more detail.

1) Choosing $\Delta f_{-}^{m}$ and $\Delta f_{+}^{m}$ : The choice of $\Delta f_{-}^{m}$ and $\Delta f_{+}^{m}$ by the coordinator dictates the way transmission capacity will be eventually allocated to the TSs. As discussed in the Introduction, an implicit auction approach, in which limited transmission capacity is allocated in the course of clearing the (multiple) markets, is chosen to deal with congestion management.

As mentioned in Section II-C, only non commerciallysensitive information, such as the cleared schedules from TSs, should be communicated between involved parties. In this context, it is proposed to allocate transmission capacity to TSs in proportion to their respective utilizations of the congested branches.

Coming back to the overloaded branch $b$, this choice suggests that the constraint (20), reflecting the share of the transmission capacity among the TSs, should be:

$$
\mathbf{s}_{b} \mathbf{p}^{m} \leq \frac{\mathbf{s}_{b} \mathbf{p}_{o}^{m}}{\sum_{m} \mathbf{s}_{b} \mathbf{p}_{o}^{m}} f_{b}^{\max }
$$

The above equation is equivalent, as can be shown by using (15) and (20), to choosing $\Delta f_{-}^{m}$ such that:

$$
\frac{\Delta f_{-}^{m}}{\sum_{m} \Delta f_{-}^{m}}=\frac{\mathbf{s}_{b} \mathbf{p}_{o}^{m}}{\sum_{m} \mathbf{s}_{b} \mathbf{p}_{o}^{m}}
$$

Similarly, for a lower limit violation, $\Delta f_{+}^{m}$ is taken as:

$$
\frac{\Delta f_{+}^{m}}{\sum_{m} \Delta f_{+}^{m}}=\frac{\mathbf{s}_{b} \mathbf{p}_{o}^{m}}{\sum_{m} \mathbf{s}_{b} \mathbf{p}_{o}^{m}}
$$

Equation (21) suggests that the more a TS is using a congested branch the more it has the right to keep on using it. 


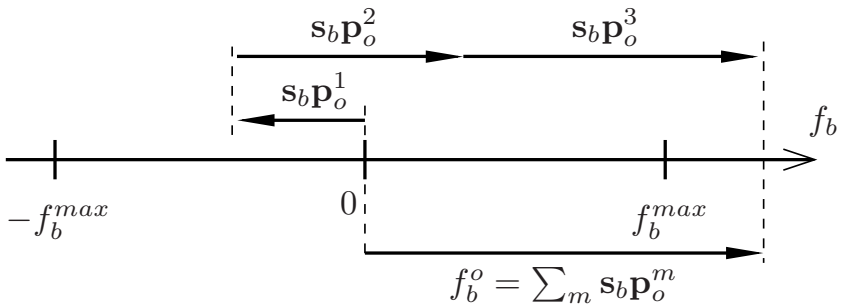

Fig. 1. Example of counterflow situation

This goes towards increasing efficiency: the more a TS uses a branch, the more this is likely to be valuable for its schedule.

On the other hand, (21) can be rewritten as

$$
\mathbf{s}_{b}\left(\mathbf{p}^{m}-\mathbf{p}_{o}^{m}\right) \leq \frac{\mathbf{s}_{b} \mathbf{p}_{o}^{m}}{\sum_{m} \mathbf{s}_{b} \mathbf{p}_{o}^{m}}\left(f_{b}^{\max }-\mathbf{s}_{b} \mathbf{p}_{o}\right)
$$

which shows that the more a TS participates in a congestion, the more it has to participate in its alleviation. This meets the objective of fairness and practical acceptability of the policy: the larger the responsibility of a TS in a flow, the larger the correction requested from this TS.

These two interpretations of (21) may look contradictory at a first glance but are mathematically equivalent owing to the choice of proportionality. The allocation rule is further discussed in Section VI-B.

2) Counterflow situations: It may happen that the schedule of a TS creates a counterflow in an overloaded branch. This situation is depicted in Fig. 1, which refers to a case with three TSs. In the situation shown, the branch is overloaded but the contribution $\mathbf{s}_{b} \mathbf{p}_{o}^{1}$ of the first TS is in the opposite direction with respect to the power flow $f_{b}^{o}$. Clearly, this TS reduces the overload caused by the other two TSs.

It would not be fair to impose a congestion management constraint to a TS that contributes with such a counterflow, since the latter in fact reduces the overload created by the other TS schedules. On the contrary, the counterflow leaves more room for the transactions of the other TSs, which is good from the market viewpoint. Hence, when allocating the available capacities among TSs, it is reasonable to let unconstrained the TSs that cause counterflows and share the effort among the other TSs. To this purpose, for a branch with an upper limit violation (12) it suffices to use (14) with the sums extending only over the schedules with positive contributions $\mathbf{s}_{b} \mathbf{p}_{o}^{m}$. Similarly, for a branch with a lower limit violation (17), only the schedules with a negative contribution are considered when using (18).

As will be explained in Section III-C, iterations are performed between market clearings by the TSs, on one hand, and Transmission allocation by the coordinator, on the other hand. If the TS producing the counterflow is not requested to change its schedule, there is no reason for that TS to depart from its optimum, and it will keep on contributing with the same counterflow. On the other hand, if the handling of another branch overload requires the TS to change its schedule, it may happen that its counterflow is decreased. In this case, at the next iteration, the branch will still be overloaded and through a new application of $(14,18)$ the other TSs will be requested

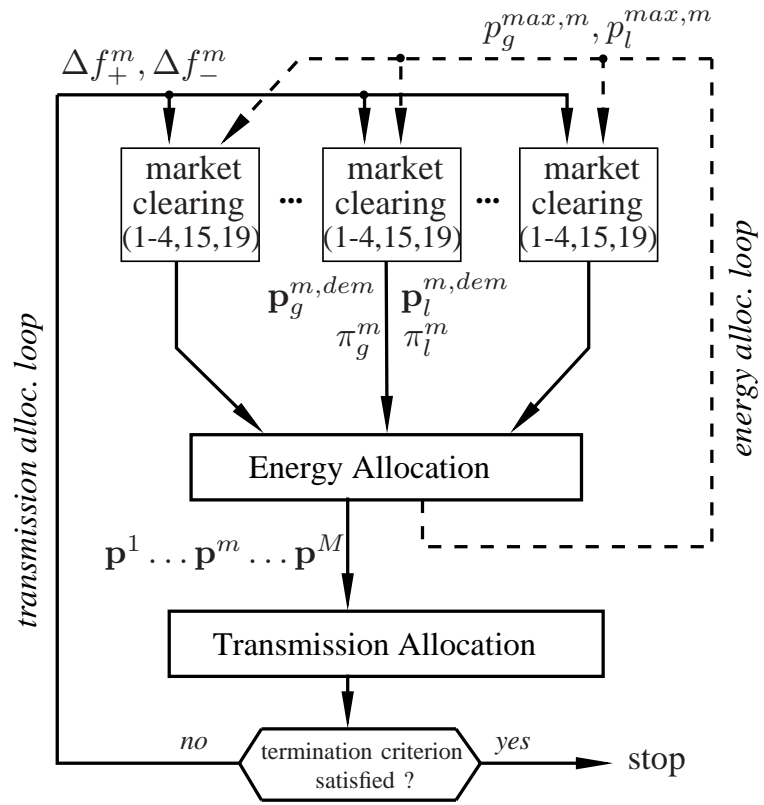

Fig. 2. Flowchart of the iterative Energy and Transmission allocations

to contribute more towards its alleviation. Obviously, if a TS stops counterflowing, it enters the congestion management procedure as the other TSs.

3) Handling multiple congestions: After a branch overload has been handled as described above, it should be prevented from taking place again in subsequent iterations, for instance when other branch overloads are handled. To this purpose, the inequality constraints $(14,18)$ stemming from previous congestion managements should remain in effect when dealing with new congestions ${ }^{2}$. Of course, for the formerly congested branches, the constraints will allow using the remaining part of the available capacity (i.e. $\Delta f_{-}^{m}$ and $\Delta f_{+}^{m}$ are negative for such branches).

\section{Overall procedure for Energy and Transmission allocations}

As already mentioned, in the general case, iterations need to be performed between the Energy and Transmission allocation procedures. The overall procedure is outlined in Fig. 2.

The procedure starts with each TS clearing its market according to its own procedures and rules. The resulting demanded (not approved yet) schedules and corresponding offered prices are communicated to the coordinator.

The latter first deals with Energy allocation. When the received schedules are in conflict, resources are allocated as explained in Section III-A and new constraints regarding the availability of these resources are communicated to the TSs, which clear again their markets. The procedure, depicted with dashed line in Fig. 2 is repeated until the coordinator eventually receives schedules with no availability conflict; the latter are used in the Transmission allocation block.

This block performs the computations presented in section III-B and, in case of congestions, sends back the constraints

\footnotetext{
${ }^{2}$ again, note that constraints of the type (11) cannot be used because the various TSs clear their markets simultaneously
} 
$(14,18)$ to the TSs for inclusion in their market clearing. This makes up an outer loop, shown with heavy line in Fig. 2.

Before doing so, a convergence test is performed on all branches that have been involved in constraints $(14,18)$. If any power flow differs from the value at the previous iteration by more than a tolerance $\epsilon$, the algorithm proceeds with a new Energy allocation loop; otherwise the procedure is completed.

One could think of stopping the iterations as soon as the schedules resulting from the $M$ simultaneous market clearings do not lead to any new branch overload. The reason for not doing so can be seen from the following counterexample. Due to the flow it causes in branch $b$, the constraint $\mathbf{s}_{b}\left(\mathbf{p}^{m}-\mathbf{p}_{o}^{m}\right) \leq$ $-\Delta f_{-}^{m}$ is imposed to the $m$-th TS, and $\mathbf{s}_{b}\left(\mathbf{p}^{k}-\mathbf{p}_{o}^{k}\right) \leq-\Delta f_{-}^{k}$ to the $k$-th TS. Assume furthermore that when clearing its market, the $k$-th TS comes up with a schedule $\mathbf{p}_{\text {new }}^{k}$ such that its participation to the $b$-th power flow is lower than expected, i.e. $\mathbf{s}_{b}\left(\mathbf{p}_{\text {new }}^{k}-\mathbf{p}_{o}^{k}\right)<-\Delta f_{-}^{k}$ (which may happen if this TS has to satisfy other constraints as well). Then, some transmission capacity is left unused. The procedure should not stop but leave the $m$-th TS the opportunity to exploit this margin, for the sake of economic efficiency. However, if needed due to limited remaining time, the procedure could stop at an intermediate, already available, feasible schedule.

\section{Information flow during the execution of the algorithm}

It is appropriate to summarize the information disclosed and communicated between parties.

Each market participant places its bid to a number of TSs (generally, different per TS). This information is given only to the TS receiving the bid. At no point of the procedure it is revealed to any other entity.

Every time the TSs simultaneously clear their markets, they announce to the coordinator their preferred schedules and the prices they offer to the market participants. This information is made available only to the coordinator during the procedure, but it could be disclosed at the end so that interested parties can check that the coordinator has acted according to the rules.

The coordinator communicates to the TSs linear constraints relating their net bus power injections with sought changes in branch flows. The model used by the coordinator to compute those flows is in principle available to all market participants, allowing them to check that they have been properly treated during the execution of the algorithm.

\section{Simulation RESUltS ON A 15-BUS TEST SYSTEM}

\section{A. Test system}

For clarity, we illustrate the features of the proposed approach on a problem where: (i) the loads are considered fixed, i.e. only the generators are bidding, and (ii) each of the involved TSs serves the load of an area. Note that the method is generally able to handle situations where each TS serves loads dispersed throughout the whole system, or some loads place their bids simultaneously to more than one TSs.

Thus, each TS dispatches generation, located anywhere in the interconnection, so as to satisfy the load located in its area. This leads to the simple market clearing:

$$
\min _{p_{g_{i}}^{m}} \sum_{i} c_{i}^{m} p_{g_{i}}^{m}
$$

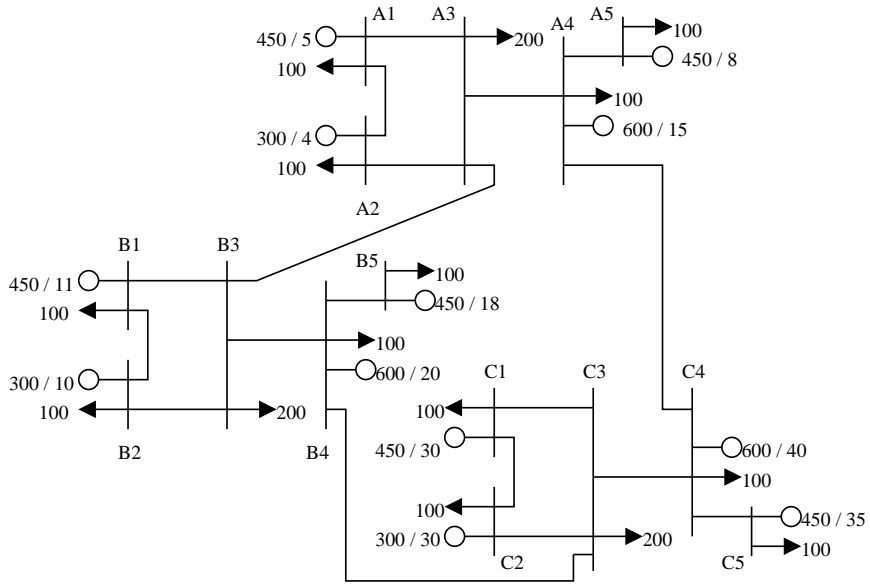

Fig. 3. 3 area test system

$$
\begin{gathered}
\text { s. t. } \quad \sum_{i} p_{g_{i}}^{m}=\sum_{j} p_{l_{j}}^{m} \\
0 \leq p_{g_{i}}^{m} \leq p_{g_{i}}^{\max , m} \\
\mathbf{s}_{b}\left(\mathbf{p}_{\mathbf{g}}^{m}-\mathbf{p}_{\mathbf{l}}^{m}-\mathbf{p}_{\mathbf{0}}^{m}\right) \leq-\Delta f_{-}^{m} \quad b=1, \ldots \\
\mathbf{s}_{b}\left(\mathbf{p}_{\mathbf{g}}^{m}-\mathbf{p}_{\mathbf{l}}^{m}-\mathbf{p}_{\mathbf{0}}^{m}\right) \geq \Delta f_{+}^{m} \quad b=1, \ldots
\end{gathered}
$$

where all symbols have been previously defined, and the last two constraints stem from the coordinator.

The 3-area 15-bus system shown in Fig. 3 is used. It consists of three 5-bus areas, each of them serving $600 \mathrm{MW}$ of load. The three areas are identical as regards the distribution of loads and the location and capacity of generators. However, they differ by the generator bids, which are the cheapest in area A and the most expensive in area $\mathrm{C}$. Each generator is assumed to make the same bid to all three TSs (i.e. $c_{i}^{A}=c_{i}^{B}=c_{i}^{C}$ ).

\section{B. Examples of iterations}

In order to provide insight on how the algorithm performs, we present hereafter the results obtained at the first three iterations of the procedure, followed by those of the final generation schedule.

At the initial point, all TSs are allowed to compete for all generators without any other constraint than (25) and (26), with $p_{g_{i}}^{\max , m}=p_{g_{i}}^{\max }, \forall m$. Obviously, this leads to all of them simultaneously demanding the cheapest generations, i.e. all TSs ask for $300 \mathrm{MW}$ from gA2 and $300 \mathrm{MW}$ from gA1. Hence, the Energy allocation procedure merely divides the available generation in equal parts, and these constraints are sent back to the TSs for them to perform new market clearings. This step is repeated, as shown by the dashed line in Fig. 2, until no two TSs compete for the same power generation. This yields the situation detailed in Table I. For each generator, Columns 1 and 2 give its name and bid ( $€ / M W h)$, Columns 3 to 5 show the power allocated to each TS, Column 6 gives its total allocated generation (i.e. the sum of Columns 3 to 5), while Column 7 shows its maximum production capacity.

At this stage, the coordinator can determine the resulting flows and check the corresponding limits. The results for the overloaded branches are given in Table II. Columns 2 to 4 
TABLE I

ITERATION 1: GENERATION ALLOCATED TO EACH TS (IN MW)

\begin{tabular}{|c|c||c|c|c|c|c|}
\hline Gen & $c_{g}$ & $p_{g}^{A}$ & $p_{g}^{B}$ & $p_{g}^{C}$ & $p_{g}^{\text {total }}$ & $p_{g}^{\text {max }}$ \\
\hline \hline gA1 & 5 & 150 & 150 & 150 & 450 & 450 \\
\hline gA2 & 4 & 100 & 100 & 100 & 300 & 300 \\
\hline gA4 & 15 & 0 & 0 & 0 & 0 & 600 \\
\hline gA5 & 8 & 150 & 150 & 150 & 450 & 450 \\
\hline \hline gB1 & 11 & 100 & 100 & 100 & 300 & 450 \\
\hline gB2 & 10 & 100 & 100 & 100 & 300 & 300 \\
\hline gB4 & 20 & 0 & 0 & 0 & 0 & 600 \\
\hline gB5 & 18 & 0 & 0 & 0 & 0 & 450 \\
\hline \hline gC1 & 30 & 0 & 0 & 0 & 0 & 450 \\
\hline gC2 & 30 & 0 & 0 & 0 & 0 & 300 \\
\hline gC4 & 40 & 0 & 0 & 0 & 0 & 600 \\
\hline gC5 & 35 & 0 & 0 & 0 & 0 & 450 \\
\hline
\end{tabular}

show the participation of each TS to each branch flow, while Columns 5 and 6 give respectively the branch flow and its limit. The last three columns of the table show by how much each TS will be requested to change each power flow in its next market clearing, according to Eq. (22). Adding together the various $\Delta f_{-}^{m}$ values of a branch yields the overload $f_{b}-f_{b}^{\text {max }}$ that has to be corrected.

It is noteworthy that TS A is obliged to decrease the flows on branches A1A3 and A2A3 by less than the other two TSs, even if all three are allocated the same power from generators gA1 and gA2. This is due to the fact that TS A serves some loads on buses A1 and A2, which makes it less responsible for the flows on those two branches.

Finally, the dash in the last row of Table II means that TS A is not requested to change its contribution to the branch flow A4C4 because it is counterflowing, as explained in Section III-B2. Indeed, TS A has a negative contribution of $-42 \mathrm{MW}$ to the final branch flow of $408 \mathrm{MW}$. The necessary power flow decrease by $408-200=208 \mathrm{MW}$ is assigned to the other two TSs, in proportion to their participation.

TABLE II

ITERATION 1: POWER FLOWS AND REQUESTED CORRECTIONS (IN MW)

\begin{tabular}{|c||c|c|c|c|c|c|c|c|}
\hline Line & TS A & TS B & TS C & $f_{b}$ & $f_{b}^{\max }$ & $\Delta f_{-}^{A}$ & $\Delta f_{-}^{B}$ & $\Delta f_{-}^{C}$ \\
\hline \hline A1A3 & 32 & 133 & 133 & 298 & 150 & 16 & 66 & 66 \\
\hline A2A3 & 18 & 117 & 117 & 252 & 150 & 8 & 47 & 47 \\
\hline B1B3 & 100 & 0 & 100 & 200 & 150 & 25 & 0 & 25 \\
\hline B2B3 & 100 & 0 & 100 & 200 & 150 & 25 & 0 & 25 \\
\hline A4C4 & -42 & 125 & 325 & 408 & 200 & - & 58 & 150 \\
\hline
\end{tabular}

This completes the first execution of the Transmission allocation loop shown with solid line in Fig. 2. At this point the TSs perform new market clearings incorporating the constraints $(27,28)$. The corresponding demanded generations are shown in Columns 3 to 5 of Table III.

What makes the TSs adjust their schedules with respect to the values in Table I is the addition of the constraints dealing with the overloaded branches. For instance, TS C is obliged to abandon most of the power it planned to obtain from generators located in system $\mathrm{A}$, in order to decrease by $150 \mathrm{MW}$ the flow it causes on the tie-line A4C4 (see Table II).

When the second iteration starts, no TS can use the power allocated to another TS at the first iteration. For example, TS A can only resort to $150 \mathrm{MW}$ from generator gA5 since the remaining $300 \mathrm{MW}$ were already allocated to TSs B and C
TABLE III

ITERATION 2: GENERATION SCHEDULE (IN MW) AFTER FIRST ITERATION OF THE ENERGY ALLOCATION LOOP

\begin{tabular}{|c|c|c|c|c|c|c|c|c|}
\hline \multirow[t]{2}{*}{ Gen } & \multirow[t]{2}{*}{$c_{g}$} & \multicolumn{3}{|c|}{ demanded by } & \multicolumn{3}{|c|}{ allocated to } & \multirow{2}{*}{$p_{g}^{\text {total }}$} \\
\hline & & TS A & TS B & TS C & TS A & TS B & TS C & \\
\hline$\overline{\text { gA1 }}$ & 5 & 125 & 63 & 0 & 125 & 63 & 0 & 188 \\
\hline $\mathrm{gA} 2$ & 4 & 100 & 73 & 74 & 100 & 73 & 74 & 247 \\
\hline gA4 & 15 & 75 & 0 & 0 & 75 & 0 & 0 & 75 \\
\hline gA5 & 8 & 150 & 110 & 0 & 150 & 110 & 0 & 260 \\
\hline$\overline{\mathrm{gB} 1}$ & 11 & $\overline{75}$ & $\overline{100}$ & $\overline{\overline{0}}$ & $\overline{75}$ & $\overline{100}$ & $\overline{0}$ & 175 \\
\hline gB2 & 10 & 75 & 100 & 100 & 75 & 100 & 100 & 275 \\
\hline gB4 & 20 & 0 & 0 & 0 & 0 & 0 & 0 & 0 \\
\hline gB5 & 18 & 0 & 154 & 426 & 0 & 119 & 331 & 450 \\
\hline$\overline{\mathrm{gC} 1}$ & $\overline{30}$ & $\overline{0}$ & $\overline{0}$ & $\overline{0}$ & $\overline{0}$ & $\overline{0}$ & $\overline{0}$ & $\overline{0}$ \\
\hline $\mathrm{gC} 2$ & 30 & 0 & 0 & 0 & 0 & 0 & 0 & 0 \\
\hline $\mathrm{gC4}$ & 40 & 0 & 0 & 0 & 0 & 0 & 0 & 0 \\
\hline $\mathrm{gC5}$ & 35 & 0 & 0 & 0 & 0 & 0 & 0 & 0 \\
\hline
\end{tabular}

(see Table I). More precisely, TS A can either keep from gA5 those $150 \mathrm{MW}$ already allocated to it or make it partly or fully available to the other TSs, depending upon the outcome of its new market clearing. Indeed, Table III shows that TS A is obliged to release part of the powers allocated to it from $\mathrm{gA} 1, \mathrm{gB} 1$ and $\mathrm{gB} 2$, in order to meet the constraints stemming from branches A1A3, A2A3, B1B3 and B2B3. It should be noted how the constraint on the tie-line $\mathrm{A} 4 \mathrm{C} 4$ has affected the market clearing solutions of TS B and even more TS C, both obliged to replace cheap generation in area $\mathrm{A}$ by more expensive in area B.

For generator gB5, the total demanded generation exceeds its capacity (see highlighted values in the table). According to the rule discussed in Section III-A, the TS making the best bid has priority. A marginal clearing price mechanism has been assumed for all three TSs. Hence, the price offered by each TS is the bid of the most expensive generator in its dispatch. In this particular case, it happens that both TS B and TS C (TS A does not ask any power from gB5) offer the same price of $18 € / \mathrm{MWh}$. Hence, according to the default rule suggested in Section III-A, the remaining capacity (in this case the whole $450 \mathrm{MW}$ available) is allocated to each TS proportionally to what it asks. Columns 6 to 8 in Table III show the quantities allocated as a result of the above decisions.

Since there was one generator with demand higher than capacity, another execution of the Energy allocation loop is performed, involving new market clearings. In the latter, the congestion management constraints remain unchanged, but the $p_{g_{i}}^{\max , m}$ bounds in (26) have been updated. For instance, TS A now sees $450-110=340 \mathrm{MW}$ available from gA5, and $450-331-119=0 \mathrm{MW}$ available from gB5. From the latter, TS B and TS C see 119 MW and 331 MW respectively.

The resulting generation schedule is given in Table IV. As can be seen, TS A has released most of the generation it had in area B in order to dispatch the less expensive that is now available in area A (gA5). As there is no conflict between demanded and available quantities, the algorithm proceeds with the Transmission allocation.

The new power flow corrections are detailed in Table V, which illustrates other features of the proposed method.

First, one can see that all the previously overloaded branches have been brought back within limits, except tie-line A4C4. The reason is that not all TSs have participated in alleviating 
TABLE IV

ITERATION 2: GENERATION ALLOCATED TO EACH TS (IN MW)

\begin{tabular}{|c|c||c|c|c|c|c|}
\hline Gen & $c_{g}$ & $p_{g}^{A}$ & $p_{g}^{B}$ & $p_{g}^{C}$ & $p_{g}^{\text {total }}$ & $p_{g}^{\text {max }}$ \\
\hline \hline gA1 & 5 & 123 & 63 & 0 & 186 & 450 \\
\hline gA2 & 4 & 103 & 73 & 107 & 283 & 300 \\
\hline gA4 & 15 & 0 & 0 & 0 & 0 & 600 \\
\hline gA5 & 8 & 340 & 110 & 0 & 450 & 450 \\
\hline \hline gB1 & 11 & 0 & 100 & 7 & 107 & 450 \\
\hline gB2 & 10 & 33 & 100 & 111 & 244 & 300 \\
\hline gB4 & 20 & 0 & 34 & 0 & 34 & 600 \\
\hline gB5 & 18 & 0 & 119 & 331 & 450 & 450 \\
\hline \hline gC1 & 30 & 0 & 0 & 0 & 0 & 450 \\
\hline gC2 & 30 & 0 & 0 & 0 & 0 & 300 \\
\hline gC4 & 40 & 0 & 0 & 0 & 0 & 600 \\
\hline gC5 & 35 & 0 & 0 & 43 & 43 & 450 \\
\hline
\end{tabular}

TABLE V

ITERATION 2: POWER FLOWS AND REQUESTED CORRECTIONS (IN MW)

\begin{tabular}{|c||c|c|c|c|c|c|c|c|}
\hline Line & TS A & TS B & TS C & $f_{b}$ & $f_{b}^{\max }$ & $\Delta f_{-}^{A}$ & $\Delta f_{-}^{B}$ & $\Delta f_{-}^{C}$ \\
\hline \hline A1A3 & 16 & 67 & 38 & 121 & 150 & -4 & -16 & -9 \\
\hline A2A3 & 10 & 70 & 70 & 150 & 150 & 0 & 0 & 0 \\
\hline B1B3 & 11 & 0 & 44 & 55 & 150 & -20 & 0 & -75 \\
\hline B2B3 & 21 & 0 & 75 & 96 & 150 & -12 & 0 & -42 \\
\hline A4C4 & 18 & 67 & 175 & 260 & 200 & 5 & 15 & 40 \\
\hline B4C3 & -18 & -67 & 382 & 297 & 200 & - & - & 97 \\
\hline
\end{tabular}

the congestion of that branch. Indeed, after the first iteration, the necessary A4C4 flow decrease of $208 \mathrm{MW}$ was assigned to TS B and C, while TS A was left unconstrained owing to the counterflow it was creating. As a matter of fact, TS B and TS $\mathrm{C}$ have decreased their contribution by the expected $208 \mathrm{MW}$ amount, but the new market clearing of TS A contributes to the branch flow with $18 \mathrm{MW}$ instead of the previous $-42 \mathrm{MW}$. This change is driven by the new constraints imposed to TS A. Therefore, the line remains overloaded by $18-(-42)=$ $60 \mathrm{MW}$, as shown in Table V. Hence, new corrections are going to be imposed, in which TS A will participate since it does no longer counterflow.

Next, it should be pointed out that for branches that were previously overloaded but are not anymore (namely, A1A3, $\mathrm{B} 1 \mathrm{~B} 3$ and $\mathrm{B} 2 \mathrm{~B} 3$ ) the remaining capacity is now shared among the TSs in proportion to their contributions to the flows. This yields the negative values of $\Delta f_{-}^{m}$ shown in the table.

Finally, a new branch (B4C3) gets overloaded and hence enters the set of constraints.

A new market clearing with these updated branch flow constraints yield the generation schedule shown in Table VI.

\section{Features of the final generation schedule}

The algorithm proceeds similarly until the congested branch flows differ by less than $\epsilon=2 \mathrm{MW}$ from their values at the previous iteration. This takes place after 5 iterations and yields the final values presented in Table VII (Columns 3 to 6).

Figure 4 shows the evolution of four of the congested branch flows through the successive iterations. The horizontal line corresponds to the branch flow limit.

For comparison purposes, a single market clearing has been considered. It consists in solving a single optimization for the whole system, with the objective of minimizing the total cost (i.e. maximizing total social welfare) while respecting branch flow limits. The resulting generations $p_{g_{i}}^{\text {sngl }}$ are provided
TABLE VI

ITERATION 3: GENERATION ALLOCATED TO EACH TS (IN MW)

\begin{tabular}{|c|c||c|c|c|c|c|}
\hline Gen & $c_{g}$ & $p_{g}^{A}$ & $p_{g}^{B}$ & $p_{g}^{C}$ & $p_{g}^{\text {total }}$ & $p_{g}^{\text {max }}$ \\
\hline \hline gA1 & 5 & 132 & 98 & 0 & 230 & 450 \\
\hline gA2 & 4 & 99 & 54 & 89 & 242 & 300 \\
\hline gA4 & 15 & 0 & 0 & 0 & 0 & 600 \\
\hline gA5 & 8 & 326 & 68 & 0 & 394 & 450 \\
\hline \hline gB1 & 11 & 10 & 100 & 23 & 133 & 450 \\
\hline gB2 & 10 & 33 & 100 & 167 & 300 & 300 \\
\hline gB4 & 20 & 0 & 0 & 0 & 0 & 600 \\
\hline gB5 & 18 & 0 & 179 & 141 & 320 & 450 \\
\hline \hline gC1 & 30 & 0 & 0 & 0 & 0 & 450 \\
\hline gC2 & 30 & 0 & 0 & 0 & 0 & 300 \\
\hline gC4 & 40 & 0 & 0 & 0 & 0 & 600 \\
\hline gC5 & 35 & 0 & 0 & 181 & 181 & 450 \\
\hline
\end{tabular}

TABLE VII

FINAL GENERATION ALLOCATION (IN MW)

\begin{tabular}{|c|c||c|c|c|c||c|c|}
\hline Gen & $c_{g}$ & $p_{g}^{A}$ & $p_{g}^{B}$ & $p_{g}^{C}$ & $p_{g}^{\text {total }}$ & $p_{g}^{\text {sngl }}$ & $p_{g}^{\text {max }}$ \\
\hline \hline gA1 & 5 & 136 & 113 & 0 & 249 & 250 & 450 \\
\hline gA2 & 4 & 98 & 56 & 96 & 250 & 250 & 300 \\
\hline gA4 & 15 & 0 & 0 & 0 & 0 & 0 & 600 \\
\hline gA5 & 8 & 324 & 58 & 0 & 382 & 300 & 450 \\
\hline \hline gB1 & 11 & 9 & 100 & 48 & 157 & 250 & 450 \\
\hline gB2 & 10 & 33 & 100 & 167 & 300 & 250 & 300 \\
\hline gB4 & 20 & 0 & 0 & 0 & 0 & 0 & 600 \\
\hline gB5 & 18 & 0 & 173 & 89 & 262 & 300 & 450 \\
\hline \hline gC1 & 30 & 0 & 0 & 0 & 0 & 0 & 450 \\
\hline gC2 & 30 & 0 & 0 & 0 & 0 & 200 & 300 \\
\hline gC4 & 40 & 0 & 0 & 0 & 0 & 0 & 600 \\
\hline gC5 & 35 & 0 & 0 & 200 & 200 & 0 & 450 \\
\hline
\end{tabular}

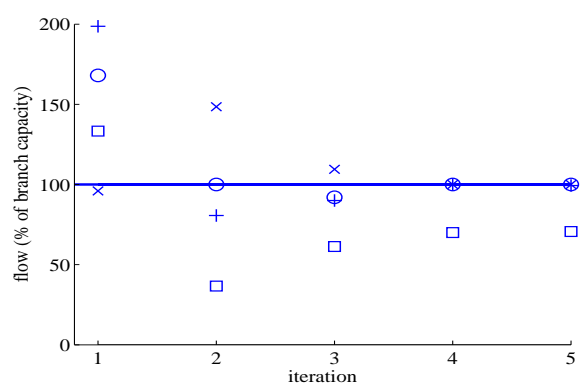

Fig. 4. Evolution of power flows with iterations: branch A1A3 is shown with,+ A2A3 with $\circ$, B1B3 with $\square$, B4C3 with $\times$

in Column 7 of Table VII, while the corresponding cost $C^{s n g l}=\sum_{i} c_{i} p_{g_{i}}^{s n g l}$ is given in Table VIII. As regards the proposed method, Columns 2 to 4 in the same table show the generation costs relative to the three TS final schedules, and Column 5 the sum of the latter costs, i.e. $C^{\text {tot }}=\sum_{m=1}^{M} C^{m}=$ $\sum_{m=1}^{M}\left(\sum_{i} c_{i} p_{g_{i}}^{m}\right)$, which corresponds to the social welfare of the entire system, obtained by the proposed method.

One can notice that with the proposed method TS A managed to allocate the cheapest schedule while TS C ended up with the most expensive one. This is due to the limited capacities of the three tie-lines $\mathrm{A} 4 \mathrm{C} 4, \mathrm{~A} 3 \mathrm{~B} 3$ and $\mathrm{B} 4 \mathrm{C} 3$ and to the fact that, during the execution of the procedure, the TSs have been obliged to reschedule their generations in order to unload congested branches. TS C has been assigned most of the effort to alleviate the overloads of these tie-lines during the execution of the algorithm (see Tables II, V).

At the final allocation no TS can further decrease its cost, by rescheduling its already allocated generation or replacing some 
TABLE VIII

FinAL GENERATION COSTS $($ IN $€ / H)$

\begin{tabular}{|c|c|c|c|c|}
\hline$C^{\text {sngl }}$ & $C^{A}$ & $C^{B}$ & $C^{C}$ & $C^{\text {tot }}$ \\
\hline 21300 & 4093 & 6467 & 11184 & 21743 \\
\hline
\end{tabular}

of it with some of the remaining available one, without causing the violation of one or more constraints. This is why some cheaper generation remains not fully exploited. For instance, TS C cannot resort to $\mathrm{gC} 1$ or $\mathrm{gC} 2$ instead of $\mathrm{gC} 5$ because shifting some generation from gC5 to $\mathrm{gC} 1$, for example, would cause the overload of one or more branches. More generally, there is no other combination involving all the generators' available quantities (i.e. not already allocated to TSs A and B) that results in a cost for TS $\mathrm{C}$ lower than $11184 € / \mathrm{h}$. There is no concern, though; TS $\mathrm{C}$ requested gC5 instead of $\mathrm{gC} 1$ or $\mathrm{gC} 2$ during the execution of the algorithm, since this allowed to allocate more interesting cheap generation outside area $\mathrm{C}$.

Expectedly, the single system-wide optimization yields a schedule with lower total cost than the proposed algorithm. This suggests that arrangements could be made between the TSs, economically profitable for all of them, such that more expensive generation is released in favor for some cheaper. It is not within the scope of this paper to simulate such arrangements but it is not incompatible with the proposed approach to let the TSs communicate with each other and exchange allocated generation quantities while clearing their markets. Of course, these inter-TS arrangements should remain consistent with the congestion alleviation obligations as well as the already allocated quantities and prices resulting from the coordinator's computations.

The cost of the system-wide optimal solution $(21300 € / \mathrm{h}$ ) is $2 \%$ lower than the total cost obtained by the proposed algorithm (21743 €/h). Let us emphasize, however, that the proposed algorithm is not aimed at minimizing the total operating cost; it should not be confused with algorithms for optimizing a single objective in a distributed manner [22], [23]. However, the fact that it yields an overall cost very close to the one obtained when handling the whole system as a single market appears to be an attractive feature. This issue is further discussed in Section VI-C.

\section{Simulation Results on IEEE RTS-96 Test System}

The algorithm was also tested on the IEEE Three-Area Reliability Test System - 1996 documented in [24]. This somewhat larger system was obtained by triplicating the One Area RTS96 system, and consists of three topologically identical 24bus systems connected with 5 tie-lines. In order to create different price areas, the marginal costs of generators have been modified with respect to [24] so that every generator in area 2 is twice as expensive as its counterpart in area 1, while the generators in area 3 are made three times as expensive as those in area 1 . Note that in spite of these price increases, area 3 still includes attractive generators compared to the other areas. Again, it was assumed that load demand is inelastic, each TS serves the loads of one area resorting to any generator, and a marginal clearing pricing mechanism is used by every
TS. The resulting scenario is interesting owing to the involved generation (re-)allocation, as shown hereafter.

TABLE IX

IEEE RTS-96 SYSTEM; INTERMEDIATE RESULTS

\begin{tabular}{|c|c|c|c|c|c|}
\hline \multirow{2}{*}{$\begin{array}{c}\text { Outer loop } \\
\text { iter. count }\end{array}$} & inner loop & \multicolumn{4}{|c|}{ cost (in $€ / h)$} \\
iterations & of TS 1 & of TS 2 & of TS 3 & total \\
\hline \hline 1 & 11 & 10457.5 & 10457.5 & 10457.5 & 31372.5 \\
\hline 2 & 1 & 10457.5 & 10457.5 & 10589.0 & 31504.0 \\
\hline 3 & 3 & 10374.7 & 10374.7 & 10587.4 & 31336.8 \\
\hline 4 & 1 & 10374.7 & 10374.7 & 10814.4 & 31563.8 \\
\hline 5 & 4 & 10280.4 & 10280.4 & 10811.7 & 31372.5 \\
\hline 6 & 1 & 10280.4 & 10280.4 & 11058.6 & 31619.4 \\
\hline 7 & 4 & 10158.2 & 10158.2 & 11056.2 & 31372.6 \\
\hline 8 & 5 & 9994.0 & 10120.0 & 11297.3 & 31411.3 \\
\hline 9 & 1 & 9995.3 & 10120.9 & 11410.1 & 31526.3 \\
\hline 10 & 2 & 9957.4 & 10091.3 & 11402.9 & 31451.6 \\
\hline 11 & 1 & 9957.5 & 10091.6 & 11417.5 & 31466.6 \\
\hline
\end{tabular}

It took 11 iterations for the procedure to converge with a tolerance $\epsilon=2 \mathrm{MW}$. Intermediate results are presented in Table IX. Each row refers to results obtained after executing the outer (Transmission allocation) loop, while the second column gives the number of inner (Energy allocation) loop executions. Columns 3 to 5 present the individual TS costs, while Column 6 shows the sum of those three individual costs.

The overall procedure can be summarized as follows. At the first iteration, network congestions are not handled yet and, since equal loads have to be served by all TSs, the cheapest generations are allocated in equal parts to each of them. This explains the identical costs shown in the table. As a result, the tie-lines of Area 3 are congested. Only TS 3 is responsible for these overloads since the other two TSs contribute with counterflows. Hence, TS 3 has to de-allocate generation in Areas 1 and 2 and replace it by more expensive in Area 3. This explains why only the cost of TS 3 increases at iteration 2 . The so released capacity is used by TS 1 and 2 at iteration 3, which explains the corresponding cost decreases. This goes with a decrease in the generation allocated to TS 1 and 2 in Area 3. Therefore, the counterflows in the above mentioned tie-lines somewhat decrease, which causes overload again. Hence, at iteration 4, TS 3 has to further correct its schedule to keep the tie-line power flows within limits. The situation is unchanged until iteration 7 when TS 1 and 2 stop counterflowing, and hence have to participate in the congestion alleviation Note that, in case of limiting time, the algorithm could even stop here as suggested at the end of Section III-C. From there on, no further line is congested and no further power flow contribution changes sign; the last iterations are devoted to satisfying the convergence criterion.

As for the 15-bus system, a comparison was carried out with a single market clearing for the whole system. The corresponding cost was found to be $31456.8 € / \mathrm{h}$, which is to be compared with the final total cost of $31466.6 € / \mathrm{h}$ obtained with the proposed procedure (see Table IX). Again, it is noteworthy that the two costs are quite close to each other; they differ by $0.031 \%$ only. 


\section{DISCUSSION}

\section{A. Incorporating bilateral trades}

It should be noted that the Energy allocation loop is optional in the proposed procedure; it is the Transmission allocation that enables the simultaneous use of the network for multiple trades. For instance, the procedure can easily accommodate bilateral trades scheduled in the spot markets ${ }^{3}$.

A bilateral trade is nothing but a schedule submitted to the coordinator by one of the sides of the trade (i.e. either the producer or the consumer plays the role of the TS). Clearly, in the Energy allocation loop the bilateral trades are always allocated as they are announced. When the feasibility of the overall schedule is checked in the Transmission allocation loop, however, it may be possible that a TS scheduling a bilateral trade is asked to decrease its flow contribution to one or several overloaded lines. In this case, it will have to cut a part of the trade. Of course, if later on during the execution of the algorithm some of this transmission capacity is made available, the TS could use it to satisfy as much of the intended trade as possible.

\section{B. On the choice of the congestion management policy}

A possibly controversial choice in the proposed algorithm, is the way the coordinator shares the use of the branches that tend to get overloaded. Economic theory would suggest that, in order to optimize the use of the whole system, each line capacity should be shared according to the economic value it has for each TS. More precisely, it was shown in [15] that at the operating point where total social welfare is maximum, all TSs equally value the use of any congested branch. Indeed, if at least one branch $b$ had a larger value for TS A than for TS $\mathrm{B}$, then the total social welfare could be further maximized by decreasing the share of the branch capacity allocated to $\mathrm{B}$ and increasing correspondingly the share allocated to A. This in turn requires computing the sensitivity of the individual TS social welfare (1) to the branch capacity assigned to that TS. Clearly, in order the above sensitivities to be compared, they must be communicated to a coordinator [14], [15].

First, it must be recalled that the method proposed in this paper does not aim at maximizing the above total social welfare but instead focuses on simultaneously optimizing multiple overlapping markets (while making the best possible use of the transmission system). Next, the proposed algorithm has been built on the premise that no TS should be asked to provide sensitive private information. In this respect, the choice of relying on the TS participation in branch flows preserves confidentiality, while it sounds reasonable, fair, and according to the test results, economically efficient. Even more, due to its simplicity, it is more transparent and could be more easily accepted by market participants and TSs.

Even if this sensitivity information was asked from the TSs, it might not be possible for the coordinator to check its validity. A mechanism should be thought to motivate the TSs to announce true sensitivity values. This can be done through

\footnotetext{
${ }^{3}$ Bilateral trades that have been scheduled in forward markets are not involved in the proposed approach (although they are taken into account when estimating the available transmission capacities).
}

TSs bidding (in explicit auctions) for individual branch transmission capacity. This would be a step back towards separate transmission and energy markets. Moreover, it may not be easy for a TS to value the use of each branch individually, in the presence of several congested branches, especially in meshed systems. Indeed, these values are much interdependent; the value of a branch for a TS would vary depending on the TS expectation to allocate the use of other branches. This passes the complications of the overlapping markets approach to the responsibility of the TS.

Clearly, the best way for allocating transmission capacity according to its real economic value for each TS (instead of doing this according to the TS intention of use) would be to have them revealing the bids that the market participants have placed to them in order for the coordinator to run an optimization problem and figure out the transmission lines economic value per TS. This would be a step towards centralization of the markets, while the proposed approach aims at allowing co-existence of separate decentralized markets.

\section{Comparison with centralized, fully integrated approach}

The direction followed in this paper is that of a decentralized approach for merging separate interconnected markets into a single large one. An alternative is that the involved entities (market participants, SOs, regulators and others) in the separate areas-markets agree to overcome the administrative and maybe political difficulties to merge into a single centrally operated system. In this case, the new central authority could clear the entire interconnection using an algorithm that collects bids from all market participants and maximizes the social welfare of the entire interconnection. Two objections may be raised at this point.

First, the willingness of all involved parties to adhere to such a central common operation may be argued. Indeed, an individual area may not want to participate into an overall social welfare maximization because this may lead to a lower social welfare locally inside this area. A set of market participants won't agree to be part of a central solution if making an arrangement between themselves is more profitable for them.

Second, whether it is preferable to operate the market in a centralized manner or coordinate multilateral trades, has been extensively discussed. It is not the intention of this paper to come up with a choice between the two, but it is worth pointing out some pros and cons of each. In principle, centralized operation mimics the old vertical organization, with the market participants' bids replacing their marginal costs and benefits. Major advantages of this approach are: (a) transmission network constraints are taken care of implicitly when clearing the energy market and (b) experience shows that it is less exposed to "gaming" by market participants. Centralized market clearing results in nodal LMPs, that is all market participants connected on the same bus pay or get paid the same price.

The choice/need for centralization stems from the difficulty to efficiently coordinate multilateral trades being simultaneously scheduled; it is not an objective by itself. On the contrary, it goes with the principle of free trading to let 


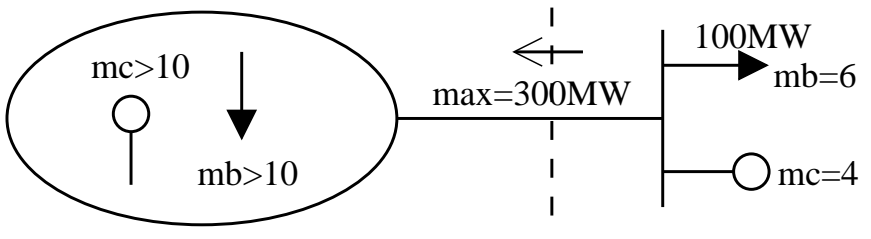

Fig. 5. Example illustrating the different shares of social welfare

market participants the option to buy and sell electric energy in the terms they agree between themselves. However, given the transmission network constraints that couple the different transactions, it is more challenging to coordinate them in a decentralized way.

The proposed decentralized scheme allows the participants to directly trade electricity in the terms they wish. Different markets could operate with different individual rules, while competition should encourage the evolution of the TSs market designs, products, software interfaces, efficiency of market clearing algorithms etc.

The co-existence of different markets allows for different ways of sharing the social welfare and for different pricing mechanisms. A generator could sell part of its production at a high price to consumers that value it a lot and another part at a lower price to consumers who are not willing to pay this much. With this price discrimination [25], neither low-paying consumers are excluded from the market, nor are cheap generators obliged to obtain low profit for energy sold to consumers that value it a lot.

The above reasoning is better illustrated through the simple example sketched in Fig. 5, where a high price area is connected to a single-bus low price area through a 300-MW transmission link. All generators of the high price area are assumed to have a marginal cost $(\mathrm{mc})$ greater than $10 € / \mathrm{MWh}$ and all loads a marginal benefit $(\mathrm{mb})$ greater than $10 € / \mathrm{MWh}$ as well. There is cheap generation available $(\mathrm{mc}=4 € / \mathrm{MWh})$ in the low price area, but it cannot be fully utilized owing to the transmission constraint. Additionally, there is some low-value load $(\mathrm{mb}=6 € / \mathrm{MWh})$ located in the low price area.

Let us first consider the case of a central market clearing resulting in nodal LMPs. If the cheap generator bids its marginal cost, it will be scheduled for a $400 \mathrm{MW}$ production at a price of $4 € / \mathrm{MWh}$, which will result in a revenue of $1600 € / \mathrm{h}$ and zero profit (it will be the marginal generator within the low price area). The generator could anticipate that the load located in the low price area is willing to pay more for energy and thus it could bid a price of $6 € / \mathrm{MWh}$. In this case the generator will be again scheduled for a $400 \mathrm{MW}$ production, but now at the price of $6 € / \mathrm{MWh}$ resulting in a revenue of $2400 € / \mathrm{h}$ and a profit of $800 € / h$. Furthermore, the cheap generator could anticipate the costs and willingness to pay of the participants located in the high price area and, thus, it could submit a bid of $10 € / M W h$. In this case the low-value load will not be served and the cheap generator will be scheduled to produce $300 \mathrm{MW}$ at a price of $10 € / \mathrm{MWh}$, resulting in a revenue of $3000 € / \mathrm{h}$ and a profit of $1400 € / \mathrm{h}$. This behavior maximizes the generator's profit under the centralized LMP-based market clearing. However, there remains some unserved load in the low price area, that is willing to pay more for energy than the marginal cost of a generator who is able to provide this energy. Social welfare of (6-4)x $100=200 € / \mathrm{h}$ is lost.

In the decentralized approach proposed in this paper, different TSs could serve the high-value and the low-value load of the example. The cheap generator can again bid its capacity at $10 € / \mathrm{MWh}$ to the high-value load and make a revenue of $3000 € / \mathrm{h}$. However, in this scheme, the generator can also place a bid in the market of the TS that serves the low-value load. The value of the generator's bid price, between 4 and $6 € / \mathrm{MWh}$, will define how the extra welfare of $200 € / \mathrm{h}$ will be shared between the generator and the load of the low price area. For instance, the cheap generator could be scheduled a $100 \mathrm{MW}$ production at $6 € / \mathrm{MWh}$ to serve the low-value load, resulting in some extra $600 € / \mathrm{h}$ revenue.

As suggested, there is a welfare equal to $\sum_{i}\left(\operatorname{prg}_{i}-m c_{i}\right)+$ $\sum_{j}\left(m b_{j}-p r l_{j}\right.$ ) (with $\operatorname{prg}_{i}$ and $\operatorname{prl}_{j}$ the price paid to the $i$-th generator and paid by the $j$-th load) that, depending on the market clearing mechanism, is to be shared between the participants. A part of this money should be withheld by the TS who clears the market in order to cover its operational costs. One can see that letting market participants the choice of TS, introduces competition among TSs to clear their markets as efficiently as possible.

To close the centralized vs. decentralized discussion, it must be emphasized that a decentralized approach like the one presented in this paper does not aim at maximizing total social welfare in the short-term, unlike what typically a centralized approach does. The former rather allows for free electricity trade according to the market participants' preferences. It is in the longer-term that a decentralized approach may be more beneficial than the centralized one, due to the market openness and the innovation it promotes. In this respect, if the shortterm results of a decentralized approach are far worse than those of the centralized one, this suggests that it is not worth being considered, since its possible longer-term benefits will not be expected to compensate for the short-term inefficient use of the energy and transmission resources. On the other hand, if a decentralized approach results in schedules with total economic value close to the optimum obtained by the centralized solution, this is a good indication that the approach under examination may be a worthy one.

\section{Conclusion And PERSPeCtives}

This paper has investigated the possibility of allowing external actors to bid in whatever market of an interconnection, thereby leading to co-existence of several overlapping markets. The procedure is based on the following premises:

- the SOs put efforts together in order to come up with and share a common network model as well as jointly operate a central coordinator;

- the various TSs can resort to different market clearing mechanisms;

- the coordination does not require the TSs to provide information that is either economically sensitive or difficult to validate (such as Lagrange multipliers).

The proposed method is another step towards creating a common electric energy marketplace in an interconnection, 
where congestion is implicitly managed in an efficient way, both from a social fairness viewpoint as well as from an engineering one. As regards the common marketplace, the proposed Energy allocation procedure allows different electricity markets to be coupled, thus offering more options to participants and more liquidity to TSs. As for congestion management, the proposed Transmission allocation procedure offers a mechanism that is fair and easy to implement, while leading to efficient use of the transmission network.

The approach has been thoroughly illustrated on small-scale examples. Although they refer, for clarity, to a simplified situation (inelastic load, all TSs using the same pricing mechanism, etc.), the approach can encompass more involved situations. Admittedly, more testings are needed before considering the proposed method for practical application. Ongoing work is in progress on several issues such as: (a) incorporating complex bid structures; (b) vulnerability to participants or TSs trying to "game" the procedure; (c) link to existing transmission pricing mechanisms and (d) possibility of reducing the number of iterations, if prohibitive.

Regarding (c), it was assumed in this work that one or several transmission pricing schemes are in effect throughout the system. The latter are expected to be reflected in the prices offered by the TSs or/and the market participants.

Regarding (d), it is recognized that with the current state of the art the proposed iterative clearing methodology would pose an important burden in the bidding-settling process and would increase the transaction costs. However, as electricity markets mature, the bidding process is expected to become routine for generators and the motive for profit will drive them to bid across multiple markets, given the relevant framework. In addition, advances in online negotiation and electronic trade using intelligent agents [26], [27] are likely to wipe out the increased time requirements and transactions costs of the proposed iterative scheme.

\section{REFERENCES}

[1] D. Kirchen, G. Strbac, "Fundamentals of Power System Economics", John Wiley and Sons, Ltd, 2004.

[2] BELPEX, APX, Powernext, "Trilateral Market Coupling: Algorithm Appendix", March 2006, available online:

http://www.belpex.be/uploads/media/Algorithm_Appendix_v4.6_PPOadaptations_final.pdf

[3] "Development and Implementation of a Coordinated Model for regional and Inter-regional Congestion Management", ETSO-EuroPex Final Report, January 2009 ,

http://www.entsoe.eu/resources/publications/etso/congestionmanagement/

[4] F.C. Schweppe, M.C. Caramanis, R.D. Tabors, R.E. Bohn, Spot Pricing of Electricity, Kluwer Academic Publishers, 1988.

[5] R.D. Christie, B.F. Wollenberg, I. Wangensteen, "Transmission Management in the Deregulated Environment", Proceedings of the IEEE, vol. 88, is. 2, pp. 170 - 195, Feb. 2000.

[6] O. Gjerde, J. Bogas, A. DiCaprio, O.B. Fosso, S. Cisneiros, M. Uusitalo, "Congestion management: The system operators challenge to balance transmission transfer capacity with an acceptable security level", Proc. CIGRE/IEEE PES International Symposium, pp. 120-127, 2005.

[7] "Implementation Study: A report for the MoU signatories on the design of the market coupling solution in the Central West European (CWE) region, by the CWE MC Project", August 2008, available online: http://www.apxgroup.com/uploads/media/Implementation_Study_with _Logo_ta.pdf

[8] L. Vandezande, L. Meeus, R. Belmans, "The next step in the Central Western European electricity market: cross-border balancing", Bourses d'énergie, no. 1, pp. 19-24, March 2008.
[9] L. Meeus, R. Belmans, "Is the prevailing wholeshale market design in Europe and North America comparable?", Proc. IEEE Power Engineering Society General Meeting, Tampa (USA), 24-27 June 2007.

[10] Federal Energy Regulatory Commission (FERC), online: http://www.ferc.gov/market-oversight/mkt-electric/overview.asp

[11] Midwest ISO - PJM Interconnection Joint and Common Market Web site: http://www.jointandcommon.com/

[12] http://www.coreso.eu/

[13] Felix F. Wu, Pravin Varaiya "Coordinated multilateral trades for electric power networks: theory and implementation", Electrical Power and Energy Systems, vol. 21, pp. 75-102, 1999.

[14] Shangyou Hao, "Decentralized Approach to Intermarket Congestion Management", IEEE Transactions on Power Systems, vol. 20, no. 2, pp. 675-683, May 2005.

[15] Kai Liu, Yixin Ni, Felix F. Wu, T.S. Bi, "Decentralized Congestion Management for Multilateral Transactions Based on Optimal Resource Allocation", IEEE Transactions on Power Systems, vol. 22, no. 4, pp. 1835-1842, November 2007.

[16] Allen J. Wood, Bruce F. Wollenberg, Power Generation Operation and Control, 2nd. edition, John Wiley \& Sons, 1996.

[17] R. Baldick, "Variation of Distribution Factors with Loading", IEEE Transactions on Power Systems, vol. 18, no 4, pp. 1316-1323, November 2007.

[18] R. Baldick, K. Dixit, T.J. Overbye, "Empirical analysis of the variation of distribution factors with loading", IEEE Power Engineering Society General Meeting, vol. 1, pp. 221-229, June 2005.

[19] M. Liu, G. Gross, "Role of Distribution Factors in Congestion Revenue Rights Applications", IEEE Transactions on Power Systems, vol. 19, no. 2, pp. 802-810, May 2004.

[20] A. Marinakis, M. Glavic, T. Van Cutsem, "Control of phase shifting transformers by multiple transmission system operators," Proc. IEEE PowerTech Conference, Lausanne (Switzerland), July 2007, paper No 101

[21] P.R. Gribik, G.A. Angelidis, R.R. Kovacs, "Transmission access and pricing with multiple separate energy forward markets", IEEE Transactions on Power Systems, vol. 14, no. 3, pp. 865-876, August 1999.

[22] J.A. Aguado and V.H. Quintana, "Inter-Utilities Power-Exchange Coordination: A Market-Oriented Approach", IEEE Transactions on Power Systems, vol. 16, no. 3, pp. 513-519, 2001.

[23] A.G. Bakirtzis and P.N. Biskas, "A decentralized solution to the DC-OPF of interconnected power systems", IEEE Transactions on Power Systems, vol. 18 , no. 3, pp. 1007-1013, 2003.

[24] Report prepared by the Reliability Test System Task Force of the Application of Probability Methods Subcommittee, "The IEEE Reliability Test System - 1996", IEEE Transactions on Power Systems, vol. 14, no. 3, pp. 1010-1020, August 1999.

[25] W.K. Viscusi, J.E. Harrington and J.M. Vernon, "Economics of Regulation and Antitrust", The MIT Press, 4th edition, Cambridge, MA, 2005.

[26] P. Vytelingum, D. Cliff, N.R. Jennings, "Strategic bidding in continuous double auctions", Artificial Intelligence, vol. 172, issue 14, pp. 1700-1729, ISSN 0004-3702, September 2008.

[27] J. Nicolaisen, V. Petrov, L. Tesfatsion, "Market power and efficiency in a computational electricity marketwith discriminatory double-auction pricing", IEEE Transactions on Evolutionary Computation, vol. 5, issue 5, pp. $504-523$, Oct 2001.

Adamantios Marinakis graduated in Electrical and Computer engineering from the National Technical University of Athens, in 2005. Since then, he has been with the Univ. of Liège, where he got the DEA degree in 2007 and is now doing research towards Ph.D. His interests are in real-time security, congestion management, market coupling, and in particular control of large power systems by multiple actors.

Anastasios G. Bakirtzis (S'77-M'79-SM'95) received the Dipl. Eng. degree from the Department of Electrical Engineering, National Technical University, Athens, Greece, in 1979 and the M.S.E.E. and Ph.D. degrees from Georgia Institute of Technology, Atlanta, in 1981 and 1984, respectively. Since 1986 he has been with the Electrical Engineering Department, Aristotle University of Thessaloniki, Greece, where he is currently a Professor. His research interests are in power system operation, planning and economics.

Thierry Van Cutsem (F'05) graduated in Electrical-Mechanical Engineering from the University of Liège (Belgium), where he obtained the Ph.D. degree and he is now adjunct professor. Since 1980, he has been with the Fund for Scientific Research (FNRS), of which he is now a Research Director. His research interests are in power system operation, dynamics, security, simulation and optimization. 